\title{
Riesgo de Tendinitis de muñeca por movimientos repetitivos en terapeutas físicos de la clínica San Juan de Dios Lima - 2012
}

\author{
Risk of Wrist Tendinitis for repetitive movements in physical therapists at San Juan de Dios \\ Lima -2012
}

\author{
Iris Rojas ${ }^{1}$
}

http://dx.doi.org/10.21503/CienciayDesarrollo.2014.v17i1.05

\section{RESUMEN}

Objetivo: determinar el nivel de riesgo de tendinitis de muñeca por movimientos repetitivos en terapeutas físicos de la Clínica San Juan de Dios Lima - 2012.

Metodología: Tipo Descriptivo - Explicativo y el diseño no experimental - corte transversal, con una muestra de 25 terapeutas Físicos, se aplicó el instrumento Job Strain Index (Moore \& Garg), Dinamometría y la Escala Análoga Visual de Dolor.

Resultados: Analizando las tareas de los fisioterapista con pelota, balancín, rulo y movilizaciones de abducción y aducción del miembro inferior del paciente, se concluye que el nivel de riesgo es alto en el $100 \%$ de las tareas según Job Strain Index, en la escala de análoga visual de dolor representa $80 \%$ de la población del total de dolor leve, moderado y fuerte en muñecas. Finalmente los resultados de la dinamometría son comparados según los referentes internacionales.

Conclusión: Existe evidencias suficientes para confirmar la hipótesis general planteada.

Palabras Clave: Riesgo de tendinitis de muneca, movimientos repetitivos.

\section{ABSTRACT}

Objective: To determine the risk level of wrist tendinitis for repetitive movements in physical therapists in the San Juan de Dios, Lima - 2012.

Methodology: Description-explanatory and non-experimental design - cross section, with a sample of 25 Physical Therapists, the instrument Job Strain Index (Moore \& Garg), Dynamometry and Visual Analog Scale for Pain was applied.

Results: Analyzing the tasks of the physiotherapist with ball, rocker, roller and movements of abduction and adduction of the lower limb of the patient, it is concluded that the risk level is high at $100 \%$ of the tasks as Job Strain Index, on the scale of visual analog pain represents $80 \%$ of the population of total of mild, moderate and strong wrists pain. Finally the dynamometry results are compared according to the international models.

Conclusion: There is sufficient evidence to confirm the general hypothesis.

Key words: Risk of wrist tendinitis, repetitive movements.

1 Lic. TMTF. Docente de la Escuela Academico profesional de Tecnología Médica. E-mail: ideroro@gmail.com 


\section{INTRODUCCIÓN}

Con el transcurso del tiempo, se ha venido observando en diferentes lugares, profesionales de diversas especialidades durante el desempeño de sus labores cotidianas; presentan lesiones musculoesqueléticas que disminuyen las capacidades motrices de las personas, lo que evidencia la mayor dificultad durante el desempeño normal de sus actividades, trayendo a su vez serias repercusiones en el ámbito laboral, familiar, social y personal.

Dentro de estas lesiones una de las más comunes presentes es la tendinitis, la más diagnosticada de los últimos años. Es una patología muy frecuente por las variadas formas que suelen presentarse, tales como los esfuerzos excesivos, movimientos repetitivos y traumatismo directo [3]. El movimiento repetitivo se caracteriza, por ser acciones que se efectúan de la misma manera numerosas veces, a lo largo de la jornada laboral. [2]

Con este objetivo se pretende buscar evidencia de cómo influye estas dos variables tan importantes entre movimientos repetitivos; por largos periodos de tiempo que conlleva a desarrollar el riesgo de tendinitis.

Se traza como objetivo principal:

Determinar el nivel de riesgo de tendinitis de muñeca por movimientos repetitivos en terapeutas físicos de la Clínica San Juan de Dios Lima - 2012.

Los objetivos específicos son:

- Demostrar los movimientos repetitivos en la fuerza muscular en los terapeutas físicos de la Clínica San Juan de Dios Lima - 2012.

- Evaluar la influencia de movimientos repetitivos en el dolor en los terapeutas físicos de la Clínica San Juan de Dios Lima - 2012.

\section{MATERIALES Y MÉTODOS}

Se ha utilizado como instrumento el método Job Strain Index JSI), que permite evaluar el nivel de riesgo de TME (trastorno musculo esquelético) en miembros superiores debido a la combinación de factores como esfuerzo, repetición, desviación posturales y exposición presentes en las tareas repetitivas con miembros superiores en especial manos y muñecas. Se utiliza además la dinamometría, mide la fuerza de prensión global de los músculos en contracción estática y por ello mediante la técnica de fichaje y finalmente la escala análoga visual de dolor, una regla de 10 centímetros.

La metodología para la realización del presente estudio, siguió las siguientes pautas:

Ubicación del área de trabajo en Neurología y Traumatología - Ortopédica de los terapeutas físicos de la Clínica San Juan de Dios, determinar las tareas más representativas de pelota, rulo, balancín y las movilizaciones de abducción y aducción del miembro inferior del puesto trabajo, por lo que, genera el riesgo de desórdenes musculo esqueléticos; dentro del problema laboral. Y finalmente proponer alternativas de solución (Norma básica de Ergonomía del Perú) la población fue de 25 terapeutas físicos es igual a la muestra y el principal criterio de inclusión son las edades fluctúan entre los 20 y 70 años de ambos sexos. Con la finalidad de proponer recomendaciones específicas para la población en estudio.

\section{RESULTADOS}

Los resultados del estudio, señalan según el método JSI, un nivel de riesgo ALTO en el trabajo de los fisioterapeutas del área de neurología y traumatología - ortopédica, en las tareas de pelota, rulo, balancín y movilizaciones de abducción y aducción del miembro inferior; obteniendo la valoración de puntaje mayor a 7 en un $100 \%$ de la muestra evaluada. Por lo consiguiente observamos en la tabla 1 y la figura 1 . 


\section{Tabla 1: Movimientos Repetidos (JSI)}

\begin{tabular}{|c|c|c|c|c|c|c|c|}
\hline \multicolumn{8}{|c|}{$\begin{array}{c}\text { JOB STRAIN INDEX } \\
\text { MOVIMIENTOS REPETITIVOS }\end{array}$} \\
\hline $\mathrm{TF}$ & $\begin{array}{l}\text { Intensidad del } \\
\text { esfuerzo }\end{array}$ & $\begin{array}{l}\text { \% Duración } \\
\text { del esfuerzo }\end{array}$ & $\begin{array}{l}\text { Esfuerzo } \\
\text { por } \\
\text { minuto }\end{array}$ & $\begin{array}{l}\text { Postura } \\
\text { mano - } \\
\text { muñeca }\end{array}$ & $\begin{array}{l}\text { Velocidad } \\
\text { de trabajo }\end{array}$ & $\begin{array}{c}\text { Duración } \\
\text { de la tarea } \\
\text { por día }\end{array}$ & $\mathrm{T}$ \\
\hline 25 & $>$ & $>$ & $>$ & $>$ & $>$ & $>$ & $>7$ \\
\hline \multicolumn{8}{|c|}{$J S I=I E \times D E \times E M \times H W P \times S W \times D D$} \\
\hline & Nivel & \multicolumn{4}{|c|}{ Frecuencia } & \multicolumn{2}{|c|}{ Porcentaje } \\
\hline & JSI & \multicolumn{4}{|c|}{25} & \multicolumn{2}{|c|}{$100 \%$} \\
\hline
\end{tabular}

Figura 1: Movimientos repetidos del paciente sobre la pelota

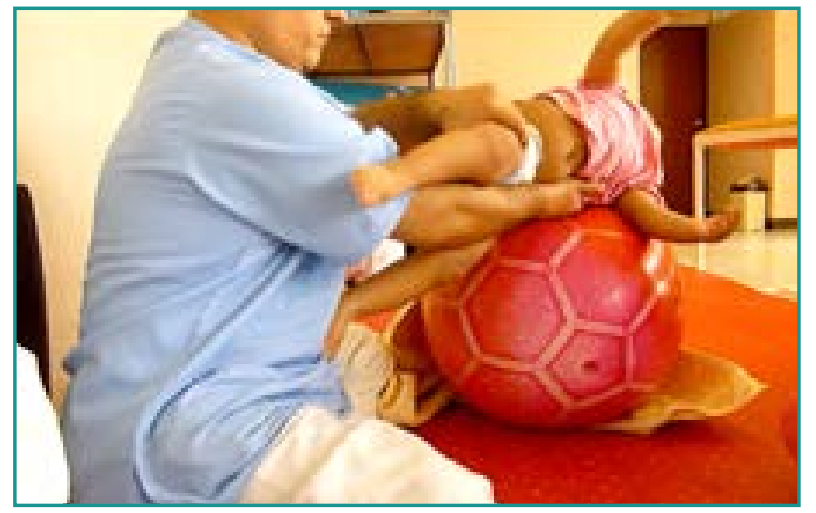

Es evidente que la fuerza muscular de la mujer es menor que la del hombre, por los diversos factores que influyen dicha situación, entre las cuales destacan la edad y el tipo de fibra muscular. Los resultados de la dinamometría son comparados según los referentes internacionales de la población, debido que en el Perú no se encuentran datos específicos sobre esto. El gráfico 1 y 2 representan los promedios de fuerza muscular en mano derecha e izquierda de mujeres y hombres, se observa que los niveles de fuerza disminuyen conforme pasa la edad.

\section{Gráfico 1: Fuerza Muscular}

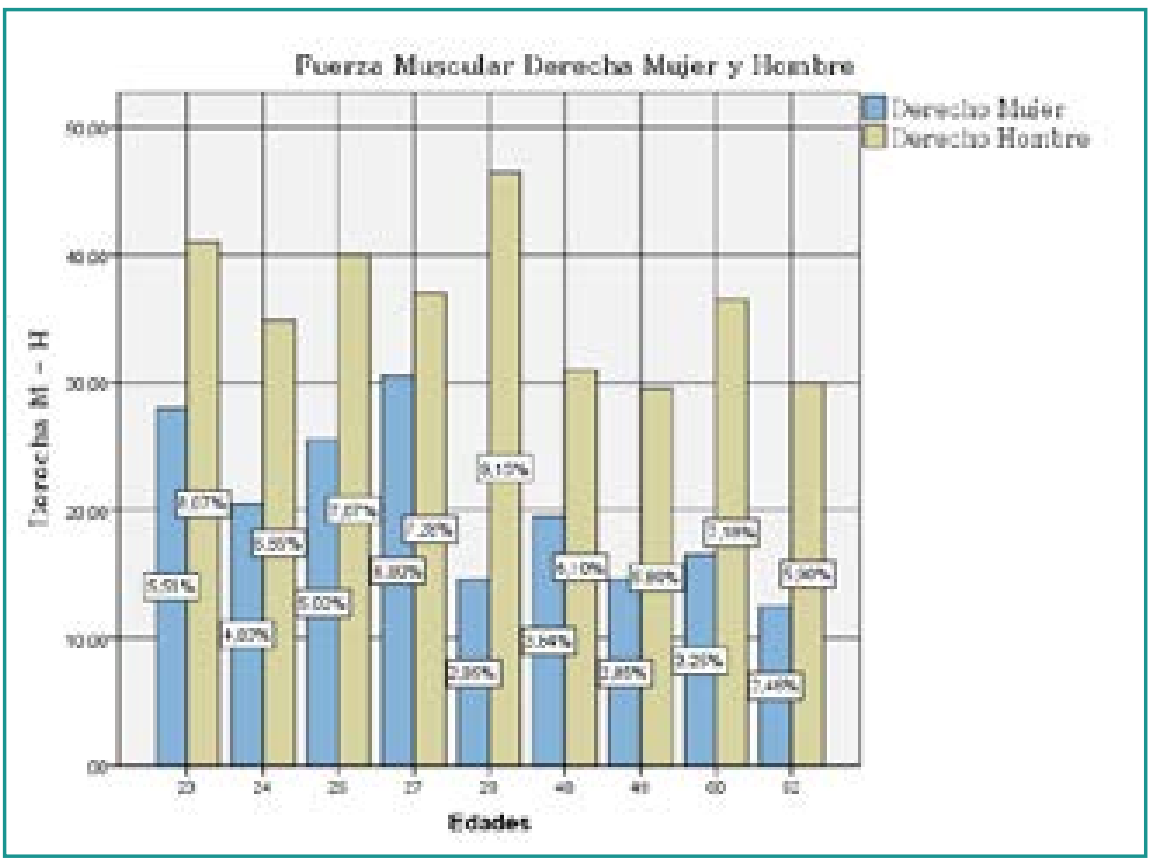

El gráfico 1; representa los promedios de fuerza muscular derecha - mujer de $35.81 \%$ del $100 \%$ de la fuerza baja y asimismo la fuerza muscular del hombre. 


\section{Gráfico 2: Fuerza Muscular}

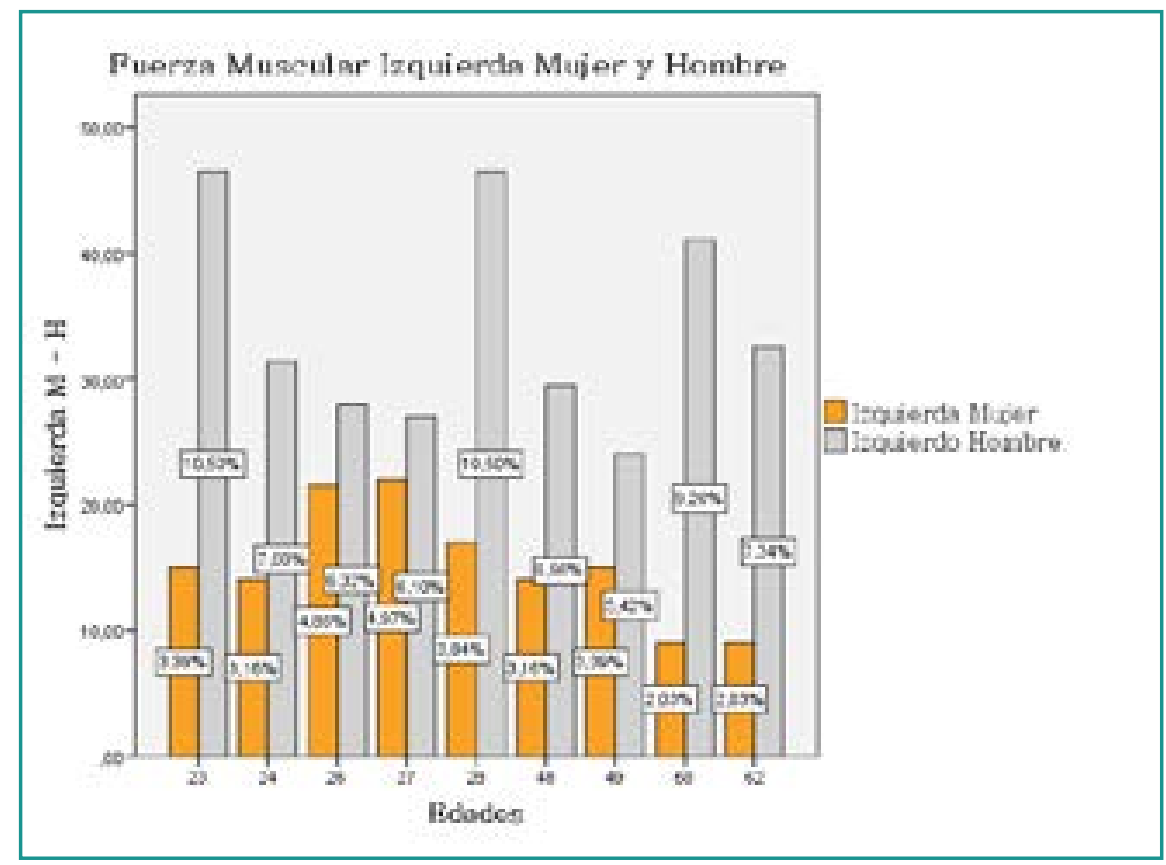

El gráfico 2; muestra los promedios de fuerza muscular izquierda - mujer de $30.83 \%$ del $100 \%$ de la fuerza baja y así mismo la fuerza muscular del hombre.

Además la Escala Visual Analógica del dolor, su aplicación es subjetiva es probablemente el componente más frecuentemente medido tanto en la práctica clínica como en la investigación de resultados posteriores. (Tabla 2)

Tabla 2: Escala del dolor

\begin{tabular}{ccc}
\hline & DOLOR & \\
\hline NIVELES & Frecuencia & Porcentaje \\
\hline $\begin{array}{c}\text { Ausencia de } \\
\text { dolor }\end{array}$ & 5 & 20 \\
\hline $\begin{array}{c}\text { Dolor leve } \\
\text { Dolor }\end{array}$ & 4 & 16 \\
moderado & 7 & 28 \\
\hline Dolor fuerte & 9 & 36 \\
\hline Total & 25 & 100 \\
\hline
\end{tabular}

En la tabla 2; se observa un $20 \%$ de ausencia de dolor, el $16 \%$ de dolor leve, $28 \%$ de dolor moderado y el $36 \%$ de dolor fuerte; el total de dolor representa un $80 \%$.

\section{DISCUSIÓN}

El nivel de riesgo ALTO en el trabajo de los fisioterapeutas del área de neurología y traumatología-ortopédica en las tareas de pelota, rulo, balancín y movilizaciones de miembro inferior en abducción y aducción. Para determinar si el trabajo es repetitivo, se considera dentro de la variedad de criterios que existen, la duración media del ciclo de trabajo inferior a 30 segundos (Putz - Anderson 1992); o, si las tareas exigen efectuar el mismo patrón de movimiento más del 50\% del tiempo del ciclo trabajo (Silverstein 1983). Según los criterios mencionados observamos que la actividad que ocupa más del $72.12 \%$ del ciclo es repetitivo porque dentro de la actividad resulta ser que el ciclo es menor a 30 segundos (total en 17 segundos), por ello ser tratada como un factor de riesgo repetitivo. Para ello la normativa Peruana (RM-375).

La evaluación de la fuerza se realiza entrevistando y la participación del terapeuta físico; preguntando el nivel de fuerza. Según el trabajador 
no le causa esfuerzo, la repetición en manos que realiza, flexo-extensión de muñeca, lo que podría llevar un acortamiento muscular por ello según la escala de BORG, se calificaría como esfuerzo débil por lo que no implica uso de fuerza directa en la actividad. Por lo tanto la mujer representa una fuerza mínima que el hombre por diversos factores por debajo de los valores normales. Según el listado de verificación establecida por la Norma Técnica Chilena (resolución -804) la falta de pausas propiamente cada hora implicaría que existe un riesgo alto de TME al no tener descanso apropiado para recuperar las extremidades. [1]

El dolor tiene relación con la fuerza muscular porque a mayor dolor menor fuerza muscular que predispone la probabilidad de desarrollar el riesgo de tendinitis de muñeca, de este modo por las características anatómicas que hacen que los tejidos sean más susceptibles a inflamarse, dependiendo del grado de exposición de movimiento.

\section{CONCLUSIONES}

- Se ha comprobado en el estudio que existe evidencias suficientes para confirmar la hipótesis general planteada: Los movimientos repetitivos de mano influyen significativamente aumentando el nivel de riesgo de tendinitis de muñeca en los terapeutas físicos de la Clínica San Juan de Dios Lima - 2012.

- Los movimientos repetitivos de mano influyen significativamente en la disminución de la fuerza muscular de prensión durante el tiempo de trabajo diario en los terapeutas físicos de la Clínica San Juan de Dios Lima $-2012$.

- Los movimientos repetitivos influyen significativamente en la aparición de dolor en los terapeutas físicos de la Clínica San Juan de Dios Lima - 2012.

- Finalmente, este estudio nos confirma que los movimientos repetitivos de mano en los terapeutas físicos de la Clínica San Juan de Dios influyen significativamente incrementando el riesgo de desarrollar o perpetuar el dolor referido en muñeca y la disminución de la fuerza muscular por tanto aumenta el riesgo de tendinitis.

\section{RECOMENDACIONES}

- Se recomienda evitar determinadas actividades o movimientos que se repiten en el rulo, pelota, balancín y abducción - aducción en el miembro inferior del niño de manera rutinaria por largos periodos de tiempo a fin de disminuir el riesgo de tendinitis de muñeca o disminuir el dolor en quienes ya lo presentan.

- El presente estudio demuestra que hay una relación proporcional entre la edad y el grado de fuerza de prensión, así mismo el grado de fuerza muscular también está en relación al sexo viéndose mayor fuerza en varones. Por lo tanto se recomienda a los terapeutas físicos en especial los casos encontrados de los jóvenes fortalecer los músculos de muñeca a fin de disminuir el riesgo de tendinitis en estos músculos.

- Por consiguiente, se debe realizar un plan de trabajo en la institución en la cual se hizo el estudio, dando pausas de tiempo de trabajo, con esta medida disminuimos el dolor referido en muñeca y el riesgo de tendinitis, de la misma manera se recomienda modificar técnicas que están implicadas en los métodos aplicados para evitar las actividades repetitivas de manera rutinaria por largos periodos de tiempo. . En tal sentido los profesionales de salud debemos dar énfasis en el higiene postural, condiciones ergonómicas y factores psico - organizacionales según el puesto de trabajo.

\section{REFERENCIAS BIBLIOGRÁFICAS}

1. Cerda Díaz, Leonidas, et al. Norma Técnica de Identificación y Evaluación de Factores de Riesgo 
Asociados a Trastornos Musculoesqueléticos Relacionados al Trabajo de Extremidades Superiores. Chile: Ministerio de Salud; 2012.

2. Cilveti Gubía, Sagrario et al. Instituto Navarro de Salud Laboral. Navarra. Salud Laboral protocolos de Vigilancia Sanitaria Específicas: Posturas Forzadas. España: Consejo Interterritorial Sistema Nacional de Salud; 2001.

3. Llaneza Alvare, Francisco Javier. La Ergonomía Forense Pruebas Periciales en Prevención del Riesgo Laboral. 2a Edición. España: Lex Nova. S. A. 2007.

4. Magazine. Prevención de los Trastornos Musculoesqueléticos de Origen Laboral. Agencia Europea para la Seguridad y la Salud en el Trabajo.2000; (10) 3.

5. Rodriguez Morales, David, et al. Enfermedades Profesionales Relacionados con los Trastornos Musculoesqueléticos: Tendinitis y Tenosinovitis del Pulgar. España: Consejo Interterritorial Sistema Nacional de Salud; 2001.

6. Sampieri Hernández et al - Metodología de la Investigación - $5^{\text {ta }}$ edición - México - Mc GRAWHILL / INTERARMERICANA EDITORES, S.A -2010 . 